\title{
REGENERAÇÃO NATURAL DE UMA ÁREA DE FLORESTA OMBRÓFILA MISTA
}

\author{
Eliana Turmina ${ }^{1 *}$, Maria Raquel Kanieski ${ }^{1}$, Ana Carolina Silva ${ }^{2}$, Pedro Higuchi ${ }^{2}$, Klerysson \\ Julio Farias ${ }^{1} \&$ Guilherme Neto dos Santos ${ }^{2}$
}

\begin{abstract}
${ }^{1}$ Universidade do Estado de Santa Catarina, Centro de Ciências Agroveterinarias, Departamento de Engenharia Florestal, Laboratório de Silvicultura e Restauração Florestal, Av. Luiz de Camões, n² 2090, Bairro Conta Dinheiro, CEP 88.520000, Lages, SC, Brasil.
\end{abstract}

${ }^{2}$ Universidade do Estado de Santa Catarina, Centro de Ciências Agroveterinarias, Departamento de Engenharia Florestal, Laboratório de Dendrologia e Fitossociologia, Av. Luiz de Camões, n 2090, Bairro Conta Dinheiro, CEP 88.520-000, Lages, SC, Brasil.

E-mails: eliana_turmina@hotmail.com (*autor correspondente); raquel.kanieski@udesc.com; carol_sil4@yahoo.com. br; higuchip@gmail.com; kjfarias.efl@gmail.com; guineto.florestal@gmail.com

Resumo: A regeneração natural se refere aos primeiros indivíduos a surgirem após um período de perturbação natural (e.g., clareiras) ou antrópica (e.g., agricultura) e é considerada como um indicador fundamental na avaliação de modelos de Planos de Recuperação de Áreas Degradadas. Estas avaliações, com base na regeneração natural, geram informações sobre o restabelecimento da vegetação, bem como dos processos ecológicos associados. Este estudo teve como objetivo avaliar a regeneração natural do componente arbóreo-arbustivo em uma área com histórico de uso e manejo agrícola e pecuário. No início de 2015, a área foi destinada a compensação florestal, sendo cercada para o desenvolvimento da floresta. A avaliação da regeneração natural foi feita no primeiro (2016) e segundo (2017) anos após o isolamento da área. Foram inventariadas 22 unidades amostrais de $100 \mathrm{~m}^{2}$ cada. Em 2016, foram amostrados 260 indivíduos, distribuídos em 13 espécies, e em 2017, a quantidade de indivíduos aumentou 96\% ( $\mathrm{N}=510)$ e $31 \%(\mathrm{~N}=17)$ para o número de espécies. Entre os períodos de levantamento ocorreu aumento na densidade, riqueza e diversidade, assim como alteração no padrão de distribuição diamétrica. A ordenação dos dados através uma análise de Escalonamento Multidimensional Não-Métrico indicou diferenças florísticoestruturais entre os períodos de avaliação, confirmada pela distância de Bray Curtis e pela NPMANOVA (ANOVA multivariada não-paramétrica permutacional). Em relação às síndromes de dispersão, não ocorreu distribuição uniforme entre as síndromes, mas sim entre os períodos de levantamento. Os padrões encontrados provavelmente foram influenciados pelo histórico da área, e sugerem que com o passar do tempo houve avanços sucessionais sobre a área de colonização, porém, também indicam uma possível necessidade de plantios de enriquecimento.

Palavras-chave: área de compensação florestal; colonização; dinâmica da regeneração natural; floresta ombrófila mista.

NATURAL REGENERATION OF A MIXED SHOULDER FOREST AREA. Natural regeneration refers to the first individuals to emerge after a period of natural (e.g., clearing) or anthropic (e.g., agriculture) disturbance and is considered as a key indicator in the evaluation of Degraded Area Recovery Plan models. These assessments, based on natural regeneration, generate information on vegetation restoration as well as 
associated ecological processes. This study aimed to evaluate the natural regeneration of the tree-shrub component in an area with a history of agricultural and livestock use and management. At the beginning of 2015, the area was intended for forest compensation, being fenced for forest development. The assessment of natural regeneration was made in the first (2016) and second (2017) years after the isolation of the area. Twenty-two sample units of $100 \mathrm{~m}^{2}$ each were inventoried. In 2016, 260 individuals were sampled, distributed in 13 species, and in 2017, the number of individuals increased by $96 \%(\mathrm{~N}=510)$ and $31 \%(\mathrm{~N}=17)$ for the number of species. Between the survey periods there was an increase in density, richness and diversity, as well as a change in the diameter distribution pattern. The ordering of the data through a Non-Metric Multidimensional Scaling analysis indicated floristic-structural differences between the evaluation periods, confirmed by Bray Curtis distance and NPMANOVA (permutational nonparametric multivariate ANOVA). Regarding the dispersion syndromes, there was no uniform distribution among the syndromes, but between the survey periods. The patterns found were probably influenced by the history of the area, and suggest that over time there have been successive advances in the area of colonization, but also indicate a possible need for enrichment plantations.

Keywords: colonization; dynamics of natural regeneration; forest clearing area; mixed ombrophilous forest.

\section{INTRODUÇÃO}

Uma das principais formações vegetacionais no sul do Brasil é a formação da Floresta Ombrófila Mista (FOM) ou Floresta de Araucária. De acordo com o mapa fitogeográfico realizado por Klein em 1978, a FOM ocupava aproximadamente $42.851,56 \mathrm{~km}^{2} \mathrm{da}$ superfície ou cerca de $45 \%$ da cobertura do estado de Santa Catarina (Klein 1978), e 73.000 km² $^{2}$ (Portal ICMBio 2019) ou 50\% do estado do Paraná (Campos \& Silveira-Filho 2010). Atualmente a cobertura remanescente é de aproximadamente 26\% (Ribeiro et al. 2009) e 3\% (Ministério do Meio Ambiente 2015) da cobertura original respectivamente por estado.

A FOM, assim como a maioria das florestas do Domínio Atlântico, passou por uma grande exploração antrópica, pelas expansões agrícola e pecuária no passado e, como consequência, ocorreu elevada redução de sua área, além da geração de impactos negativos nos remanescentes, tais como a redução no porte ou número de espécies exploradas, e diminuição dos corredores ecológicos, entre outros (Hauresko et al. 2017).

Outro fator que gera a exploração das florestas ainda hoje é a instalação de empreendimentos, como as Linhas de Transmissão de Energia (LTs). As LTs servem para distribuir a energia da usina (i.e., local de geração de energia) até o seu destino final (i.e., geralmente subestações de distribuição), e para que isso ocorra as LTs percorrem longas distâncias, passando pelos meios biótico e físico e gerando diversos impactos negativos como a supressão da vegetação. Um meio de minimizar esses impactos é o uso de medidas compensatórias (Tobouti \& Santos 2014).

As medidas compensatórias tendem a compensar as perdas ambientais devido à conversão de ambientes, podendo ser entendida como um mecanismo de responsabilização do empreendedor pelos danos/impactos negativos causados ao ambiente (Santana 2018). Uma das medidas de compensação florestal é o programa de recuperação de áreas degradadas. As técnicas utilizadas nos programas de recuperação são bastante amplas, como condução da regeneração natural ou plantio de mudas, entre outras (Kageyama \& Gandara 2000), sendo que a técnica adequada a cada área depende dos níveis de degradação e fonte de propágulos (Rech et al.2015).

Neste sentido, a intensidade das alterações proporcionadas pelo homem nesses ecossistemas influencia na recuperação da floresta, fazendo com que a mesma apresente diferentes trajetórias sucessionais, observadas em toda a extensão da formação florestal, em termos de distribuição diamétrica, área basal, densidade dos indivíduos, mortalidade dos indivíduos remanescentes e, principalmente, na riqueza de espécies (Pereira $e t$ al. 2015). Assim, acompanhar o desenvolvimento da vegetação arbustiva e arbórea por meio de estudos de dinâmica é cada vez mais importante, pois permitem obter informações sobre a trajetória sucessional dos fragmentos, sendo possível observar as mudanças (Santos et al. 2012). Uma forma de acompanhar esse desenvolvimento 
é a avaliação da regeneração natural, a qual é considerada como um indicador fundamental na avaliação de modelos de Planos de Recuperação de Áreas Degradadas (PRAD), e seu estudo é considerado uma técnica de monitoramento e avaliação na recuperação de áreas (Bellotto et al. 2009).

O acompanhamento e avaliação na recuperação de áreas degradadas com base na regeneração natural geram informações sobre o desenvolvimento e restabelecimento da vegetação. Por meio desses estudos também é possível obter diversas informações sobre os processos ecológicos associados, da autoecologia, ramo da ecologia que estuda as relações das espécies com o seu meio, definindo a ação dos fatores ecológicos do meio sobre o comportamento, fisiologia e morfologia das espécies, como o estágio sucessional, composição florística e estrutural do componente arbóreo e arbustivo (Schroter \& Kirchner 1902).

Vários estudos prévios avaliaram os impactos ambientais da implantação de LTs (e.g., Pires 2005, Ferreira 2011, Pereira 2014, Turmina et al. 2018), porém, ocorre uma carência de estudos voltados ao monitoramentodessasáreas. Essefatoocorredevido ao elevado custo da atividade de monitoramento e devido à forma como a recuperação é tratada pelos órgãos públicos licenciadores e pelas empresas que contratam e executam serviços de recuperação (Brancalion et al. 2012). Muitas vezes, a recuperação é conduzida apenas para o cumprimento de demandas específicas de certificação e de licenciamento ambiental, sem que haja um efetivo comprometimento com a sustentabilidade ecológica dessas áreas (Brancalion et al. 2012). Assim, a avaliação e o monitoramento são fundamentais para redefinir a trajetória ambiental da área em processo de recuperação (Brancalion $e t$ al. 2012).

Este estudo teve como objetivo avaliar as alterações da regeneração natural do componente arbustivo-arbóreo, em área com histórico de uso e manejo agrícola e pecuário, no primeiro e segundo ano após o isolamento da área. A área foi previamente destinada à compensação florestal referente à implantação de LT $525 \mathrm{kV}$ Salto Santiago - Itá - Nova Santa Rita, em uma área de FOM no Sul do Brasil.

\section{MATERIAL E MÉTODOS}

A área estudada está inserida na formação de FOM, próxima às margens da BR-280, na Unidade de Conservação da Estação Ecológica (ESEC) Mata Preta (Figura 1), divisa entre os estados do Paraná e Santa Catarina, no município de Abelardo Luz, estado de Santa Catarina (26 $28^{\prime}$ S e $52^{\circ} 09^{\prime} \mathrm{W}$ ), com altitude de $776 \mathrm{~m}$ (Climate-data.org 2017). A classificação climática na região é Cfb (Alvares et al. 2013), com temperatura média de $17,3^{\circ} \mathrm{C}$ e ocorrência de geadas frequentes nos meses frios; a precipitação média anual é de $2.216 \mathrm{~mm} / \mathrm{ano}$, com chuvas bem distribuídas durante o ano (Climatedata.org 2017).

Até outubro de 2014, o local de estudo estava em posse de proprietário particular, com histórico de manejo e uso agrícola e pecuário. Uma área de 4,28 ha, foi destinada à compensação florestal, referente à LT 525 kV Salto Santiago - Itá - Nova Santa Rita. Com o objetivo de favorecer a regeneração natural e evitar a entrada de animais domésticos, no período entre novembro de 2014 a janeiro de 2015, foram realizadas as primeiras atividades para a recuperação do local: revolvimento do solo e o cercamento da área. Antes do isolamento a área não apresentava espécies arbóreas e arbustivas, devido ao seu uso destinado ao cultivo de agrícola.

Para verificar a colonização na área em processo de regeneração natural foram realizados dois levantamentos: o primeiro em abril de 2016 (aproximadamente um ano após o cercamento da área) e o segundo em abril de 2017 (aproximadamente dois anos após o cercamento da área). Para isso, foram alocadas 22 unidades amostrais (UA) permanentes distribuídas de forma sistemática, espaçadas em $20 \times 50 \mathrm{~m}$, sendo que cada UA abrangeu uma área amostral de $100 \mathrm{~m}^{2}(10 \times 10$ $\mathrm{m})$, totalizando $2200 \mathrm{~m}^{2}$ de área amostral. Dentro das UAs, foram inventariados todos os indivíduos arbóreos e arbustivos adultos com diâmetro à altura do peito (DAP; i.e., 1,30 $\mathrm{m}$ acima do solo) maior ou igual a $5 \mathrm{~cm}$. Para o levantamento dos indivíduos arbóreos e arbustivos jovens, considerados aqueles com mais de 1,5 m de altura e com menos de $5 \mathrm{~cm}$ de DAP, foi realizada a subdivisão da UA de $100 \mathrm{~m}^{2} \mathrm{em}$ quatro unidades de $25 \mathrm{~m}^{2}$ ( $5 \times 5 \mathrm{~m}$ ). Foi selecionado, de forma aleatória, um dos quatro quadrantes, em cada UA, totalizando $550 \mathrm{~m}^{2}$ amostrados para o levantamento dos indivíduos arbóreos e arbustivos 


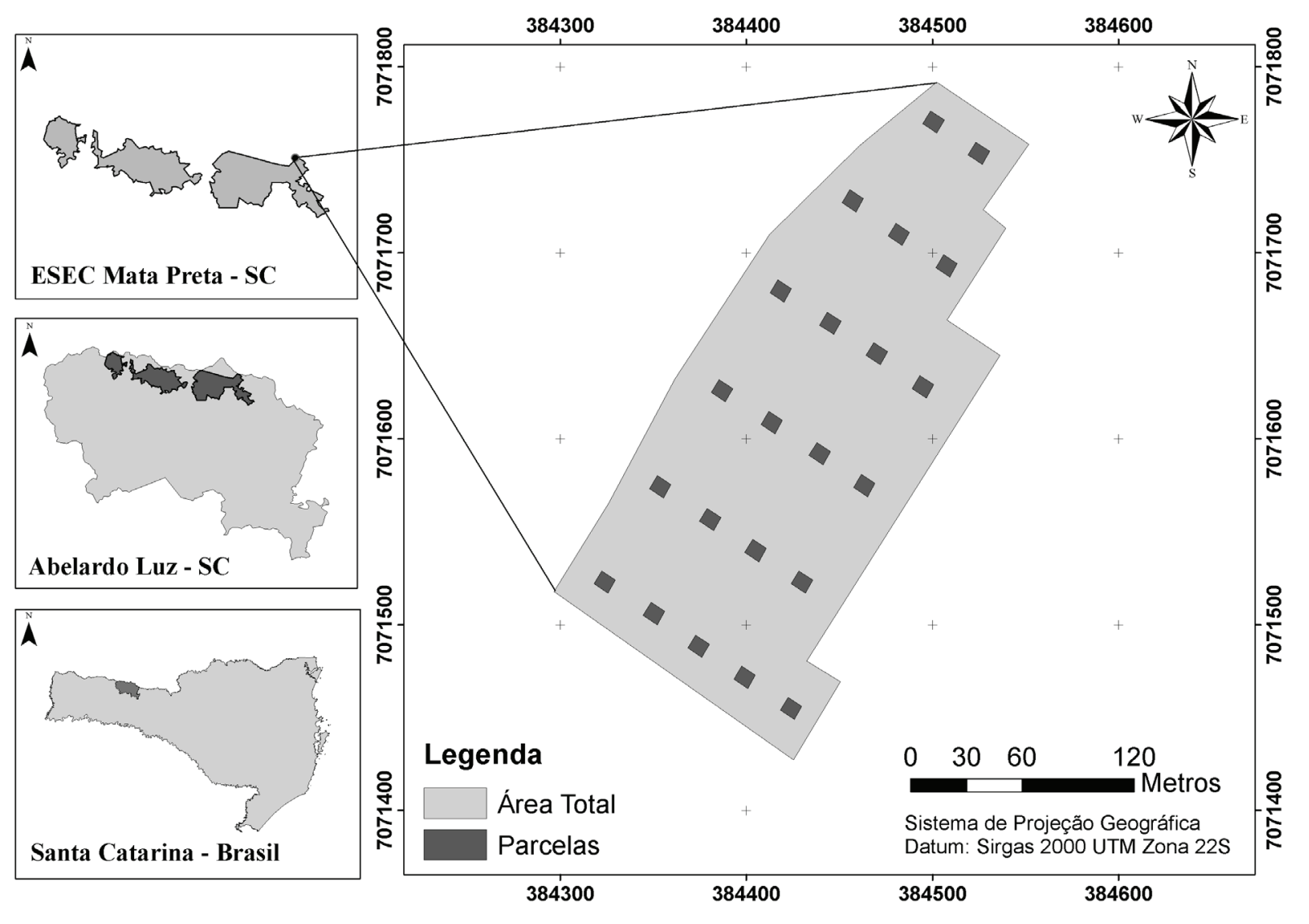

Figura 1. Localização da área em processo de regeneração natural no município de Abelardo Luz, estado de Santa Catarina, na Estação Ecológica Mata Preta, com a distribuição das parcelas de estudo.

Figure 1. Location of the area undergoing natural regeneration in the municipality of Abelardo Luz, state of Santa Catarina, at the Mata Preta Ecological Station, with the distribution of the study plots.

jovens. Salienta-se que a classificação de plantas em adultas e jovens no levantamento, não trata da classificação da idade das plantas e sim uma nomenclatura utilizada pela classificação pelos critérios de inclusão altura e DAP.

A classificação taxonômica das espécies foi feita de acordo com o sistema APG IV (Angiosperm Phylogeny Group 2016). Os materiais botânicos testemunhos foram herborizados, catalogados e incorporados ao Herbário Lages da Universidade do Estado de Santa Catarina (LUSC). A classificação da síndrome de dispersão de propágulos de cada espécie florestal nativa foi realizada segundo a metodologia de Van der Pijl (1982) e a classificação do grupo ecológico foi realizada com base em Budowski (1970).

As suficiências das amostragens dos componentes adulto e jovem, para ambos os períodos de avaliação, foram determinadas por curvas de acumulação de espécies, realizadas pelo método de aleatorização, com 1000 permutações.
Para verificar a existência de associação entre os padrões de síndrome de dispersão dos jovens e adultos, foram realizados testes de qui-quadrado $\left(X^{2}\right)$, aplicados a tabelas de contingência. Mudanças nas participações relativas das síndromes ao longo do tempo foram verificadas por meio de teste de proporção. A diversidade do componente regenerativo foi avaliada pelo índice de Shannon (H') e a dominância, pela equabilidade de Pielou (J). Os valores de $\mathrm{H}^{\prime}$ entre as avaliações com diferentes períodos de tempo foram comparados aos pares por meio do teste $t$ de Hutcheson, com $t$ tabelado de 1,64.

Para as demais análises (i.e., riqueza com número de indivíduos fixo, distribuição diamétrica e Escalonamento Multidimensional NãoMétrico), os componentes jovem e adulto foram considerados de forma conjunta. A riqueza entre as avaliações foi comparada por meio do método de rarefação, com número de indivíduos fixo, o qual é definido na curva pelo levantamento que obteve 
o menor número de indivíduos. No presente caso, a comparação da riqueza foi realizada no ponto de 260 indivíduos. Essa curva foi construída por meio do método de aleatorização, com 1000 permutações. Já a diferença florístico-estrutural entre as avaliações (2016 e 2017) foi avaliada por meio de matrizes de abundância e da distância de Bray-Curtis, em uma ordenação multivariada do tipo Escalonamento Multidimensional NãoMétrico (NMDS), e da Análise de Variância Multivariada Não Paramétrica Permutacional (NPMANOVA) com 999 permutações, considerando as matrizes de abundâncias, Bray-Curtis como medida de distância florístico-estrutural e o ano de monitoramento (2016 e 2017) como fator. A NPMANOVA permite testar se os grupos definidos pela NMDS são de fato estatisticamente diferentes entre si.

Para a representação da frequência diamétrica da comunidade em ambos os períodos de avaliação, foram construídos histogramas de frequência. Para

a)

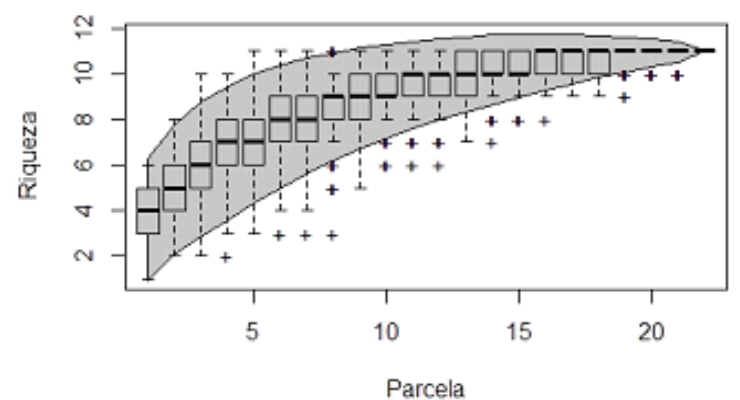

c)

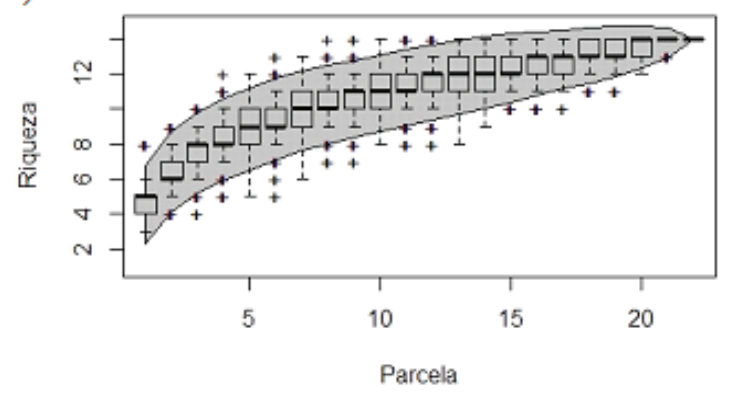

isso, foi adotado o método empírico, que consiste na determinação do número de classes pelo avaliador. Foram definidas oito classes de diâmetro para cada período de avaliação, sendo que o primeiro teve 1,38 $\mathrm{cm}$ de amplitude de classe, e o segundo, $2,10 \mathrm{~cm}$. Todas as análises foram realizadas utilizando-se planilha eletrônica e a linguagem de programação estatística R (R Development Core Team 2018), com o pacote vegan (Oksanen et al. 2018).

\section{RESULTADOS}

A curva de acumulação de espécies, para ambos os períodos de levantamento, demonstrou que a amostragem foi suficiente para caracterizar a composição florística dos indivíduos jovens e adultos da área analisada, cabendo ressaltar que as curvas não atingiram a assíntota, mostrando uma tendência ao aumento de espécies. No primeiro levantamento dos indivíduos jovens, realizado em 2016 (Figura 2a), com o acréscimo de

b)

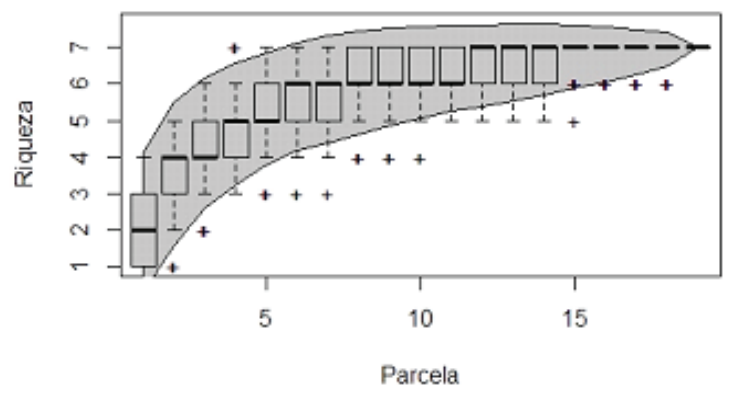

d)

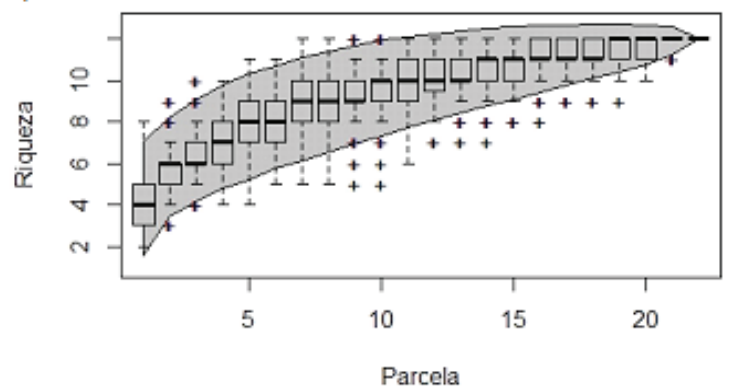

Figura 2. Curva de acumulação de espécies dos indivíduos jovens e adultos, em área em processo de recuperação no município de Abelardo Luz, estado de Santa Catarina, Brasil, sendo que os boxplots dentro da curva representam a variação da riqueza em função do desvio padrão. a = levantamento de indivíduos jovens em 2016; b = levantamento de indivíduos adultos em 2016; c = levantamento de indivíduos jovens em 2017; d = levantamento de indivíduos adultos em 2017.

Figure 2. Species accumulation curve of young and adult individuals in an area under recovery in the municipality of Abelardo Luz, Santa Catarina State, Brazil, and the boxplots within the curve represent the variation of richness as a function of standard deviation. a survey of young individuals in 2016; $b=$ survey of adult individuals in 2016; c = survey of young individuals in 2017; $d$ = survey of adult individuals in 2017. 
aproximadamente $5 \%$ da área amostrada, houve um incremento de 0,04 espécies $(0,36 \%$ da riqueza total), e para os adultos, no mesmo ano (Figura $2 b)$, incremento de 0,05 espécies $(0,71 \%$ da riqueza total). No segundo levantamento dos indivíduos jovens, em 2017 (Figura 2c), com o acréscimo de aproximadamente $5 \%$ da área amostrada, houve um incremento de 0,23 espécies $(1,64 \%$ da riqueza total), e de 0,13 espécies ( $1,08 \%$ da riqueza) para os indivíduos adultos de 2017 (Figura 2d).

No levantamento realizado em 2016, foram inventariados 260 indivíduos arbóreos e arbustivos pertencentes a 13 espécies, três famílias e sete gêneros (Tabela 1). Do total de indivíduos inventariados, 201 (77,3\%) eram jovens e 59 (22,7\%) adultos. Os 201 jovens pertenciam a 11 espécies e cinco gêneros e os 59 adultos pertenciam a sete espécies e quatro gêneros. Em 2017, foram inventariados 510 indivíduos arbóreos e arbustivos (293 ou $57,5 \%$ jovens e 217 ou $42,5 \%$ adultos), pertencentes a 17 espécies, três famílias e oito gêneros. Os 293 jovens pertenciam a 14 espécies e seis gêneros e os 217 adultos pertenciam a 12 espécies e sete gêneros (Tabela 1). Entre os levantamentos, ocorreu um aumento de $96 \%$ no número total de indivíduos (jovens e adultos), além do aumento da riqueza.

Em ambos os levantamentos, $100 \%$ dos indivíduos foram classificados como espécies pioneiras, com predominância da anemocoria. Houve destaque para a família Asteraceae, com 10 e 13 espécies, respectivamente no primeiro e segundo levantamentos. As espécies Baccharis microdonta e Baccharis dracunculifolia (Asteraceae) apresentaram o maior número de indivíduos (Tabela 1).

A comparação da riqueza de espécies, resultante da curva de rarefação com o número de indivíduos fixo (Figura 3), indicou diferença nos valores de riqueza entre as duas avaliações (2016 e 2017), ocorrendo um aumento no número de espécies ao longo do tempo, além das curvas não terem atingido a assíntota, mostrando uma pequena tendência ao aumento do número de espécies na

Tabela 1. Número de indivíduos e classificação quanto ao grupo ecológico (Budowski 1970) e síndrome de dispersão (Van der Pijl 1982) das espécies amostradas dos indivíduos jovens e adultos em área em processo de recuperação no município de Abelardo Luz, estado de Santa Catarina, Brasil. GE = Grupo ecológico, $\mathrm{P}$ = Pioneira; $\mathrm{SD}$ = Síndrome de dispersão; Zoo = Zoocórica; Ane = Anemocórica; Auto = Autocórica; No RG = Número do registro da espécie no Herbário Lages da Universidade do Estado de Santa Catarina.

Table 1. Number of individuals and classification by ecological group (Budowski 1970) and dispersal syndrome (Van der Pijl 1982) of the sampled species of young and adult individuals in an area undergoing recovery in the municipality of Abelardo Luz, Santa Catarina state, Brazil. GE = Ecological group, $P=$ Pioneer; $S D=$ Dispersion Syndrome; Zoo = zoochoric; Ane $=$ Anemochoric $;$ Auto $=$ Autochoric $; N^{\circ} R G=$ Registration number of the species in the Lages Herbarium of Santa Catarina State University.Herbarium.

\begin{tabular}{|c|c|c|c|c|c|c|c|c|}
\hline \multirow{2}{*}{ Nome Científico } & \multirow{2}{*}{ Família } & \multicolumn{2}{|c|}{2016} & \multicolumn{2}{|c|}{2017} & \multirow{2}{*}{ GE } & \multirow{2}{*}{ SD } & \multirow{2}{*}{ No RG } \\
\hline & & Jovem & Adulto & Jovem & Adulto & & & \\
\hline $\begin{array}{l}\text { A ustro e up a to rium } \\
\text { inulaefolium } \\
\begin{array}{l}\text { R.M.King \& H.Rob. } \\
\text { (Kunth) }\end{array}\end{array}$ & Asteraceae & 2 & & & & $\mathrm{P}$ & Ane & - \\
\hline $\begin{array}{l}\text { Baccharis cf. caprariifolia } \\
\text { DC. }\end{array}$ & Asteraceae & 4 & & & 1 & $\mathrm{P}$ & Ane & 9122 \\
\hline $\begin{array}{l}\text { Baccharis dracunculifolia } \\
\text { DC. }\end{array}$ & Asteraceae & 54 & 12 & 42 & 46 & $\mathrm{P}$ & Ane & 9121 \\
\hline Baccharis leucopappa DC. & Asteraceae & & & 1 & & $\mathrm{P}$ & Ane & 9149 \\
\hline Baccharis microdonta DC. & Asteraceae & 78 & 17 & 85 & 64 & $\mathrm{P}$ & Ane & 9120 \\
\hline Baccharis montana DC. & Asteraceae & 6 & 5 & 17 & 23 & $\mathrm{P}$ & Ane & 9119 \\
\hline Baccharis oxyodonta DC. & Asteraceae & & & 51 & 3 & $\mathrm{P}$ & Ane & 9118 \\
\hline Baccharis punctulata DC. & Asteraceae & 13 & & & & $\mathrm{P}$ & Ane & - \\
\hline Baccharis semiserrata DC. & Asteraceae & 35 & 12 & 20 & 40 & $\mathrm{P}$ & Ane & 9113 \\
\hline
\end{tabular}

Tabela 1. Continua na próxima página...

Table 1. Continued on next page... 
Tabela 1. ...Continuação

Table 1....Continued

\begin{tabular}{|c|c|c|c|c|c|c|c|c|}
\hline \multirow{2}{*}{ Nome Científico } & \multirow{2}{*}{ Família } & \multicolumn{2}{|c|}{2016} & \multicolumn{2}{|c|}{2017} & \multirow{2}{*}{ GE } & \multirow{2}{*}{ SD } & \multirow{2}{*}{ No $\mathbf{R G}$} \\
\hline & & Jovem & Adulto & Jovem & Adulto & & & \\
\hline Baccharis uncinella DC. & Asteraceae & 2 & & 6 & & $\mathrm{P}$ & Ane & 9117 \\
\hline $\begin{array}{l}\text { Chromolaena laevigata } \\
\text { (Lam.) R.M.King \& H.Rob. }\end{array}$ & Asteraceae & 3 & & 1 & 1 & $\mathrm{P}$ & Ane & 9116 \\
\hline $\begin{array}{l}\text { Chromolaena pedunculosa } \\
\text { (Hook. \& Arn.) R.M.King \& } \\
\text { H.Rob. }\end{array}$ & Asteraceae & & & 1 & & $\mathrm{P}$ & Ane & 9115 \\
\hline Mimosa scabrella Benth. & Fabaceae & & 2 & & 4 & $\mathrm{P}$ & Auto & 9319 \\
\hline $\begin{array}{l}\text { Raulinoreitzia tremula } \\
\text { (Hook. \& Arn.) R.M.King \& } \\
\text { H.Rob. }\end{array}$ & Asteraceae & 3 & & 10 & & $\mathrm{P}$ & Ane & 9114 \\
\hline Solanum mauritianum Scop. & Solanaceae & & 10 & 6 & 22 & $\mathrm{P}$ & Zoo & - \\
\hline Solanum variabile Mart. & Solanaceae & & & 1 & & $\mathrm{P}$ & Zoo & 9112 \\
\hline Solidago chilensis Meyen & Asteraceae & & & 6 & 1 & $\mathrm{P}$ & Ane & 9111 \\
\hline $\begin{array}{l}\text { Vassobia breviflora (Sendtn.) } \\
\text { Hunz. }\end{array}$ & Solanaceae & 1 & 1 & & 5 & $\mathrm{P}$ & Zoo & - \\
\hline $\begin{array}{l}\text { Vernonanthura tweediana } \\
\text { (Baker) H.Rob. }\end{array}$ & Asteraceae & & & 46 & 7 & $\mathrm{P}$ & Ane & 9123 \\
\hline Total & & 201 & 59 & 293 & 217 & & & \\
\hline
\end{tabular}

área avaliada.

O acréscimo da riqueza florística favoreceu o aumento dos valores do índice de diversidade (Tabela 2). Já os valores do índice de equabilidade de Pielou indicaram desuniformidade na distribuição dos indivíduos entre as espécies e predominância de dominância ecológica, exceto para o caso dos adultos no primeiro inventário $(J=0,87$ ) (Tabela 2$)$.

A ordenação através do NMDS indicou mudanças florístico-estruturais do componente vegetacional das parcelas entre 2016 e 2017 (Figura 4). A ordenação foi adequada para interpretação, uma vez que o valor de estresse foi inferior a 0,2 (stress $=0,17$ ), significando que a mesma não foi arbitrária. De acordo com a medida de distância de Bray Curtis $(0,50)$, também pode-se verificar distinções, que é confirmada pela NPMANOVA $\left(\mathrm{F}_{1,42}=7,41, p<0,001\right)$.

Em ambos os períodos de levantamento, apesar da predominância de indivíduos de espécies classificadas como anemocóricas, a distribuição dos mesmos entre os estratos de indivíduos jovens e adultos não ocorreu de forma uniforme (Tabela 3). Porém, quando se compara a distribuição das síndromes de dispersão entre os levantamentos, por meio do teste de proporção, não foi observada

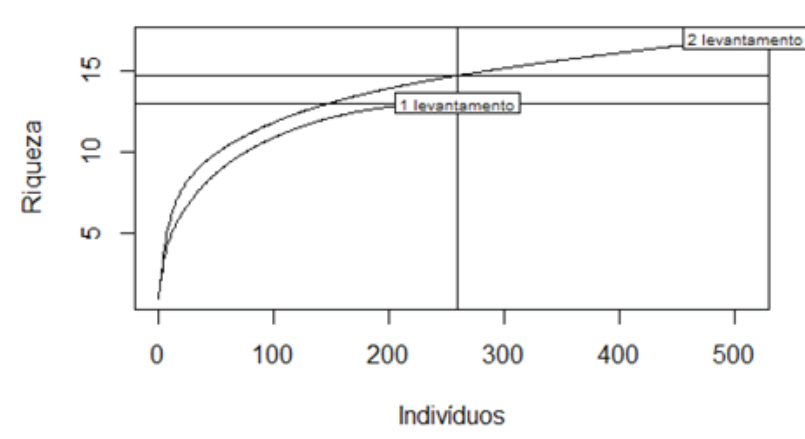

Figura 3. Curvas de rarefação para todos os indivíduos amostrados em área em processo de recuperação em 2016 e 2017, no município de Abelardo Luz, estado de Santa Catarina, Brasil.

Figure 3. Rarefaction curves for all individuals sampled in an area undergoing recovery in 2016 and 2017, in the municipality of Abelardo Luz, state of Santa Catarina, Brazil.

diferença, ou seja, as síndromes de dispersão seguiram a mesma distribuição proporcional.

$\mathrm{Na}$ avaliação das distribuições diamétricas realizadas em 2016 e 2017, é possível observar, de forma geral, a redução no número de indivíduos com o aumento da classe diamétrica (Figura 5). Porém, a primeira avaliação apresentou a distribuição diamétrica unimodal, com forte assimetria positiva. Já modelo de distribuição 
Tabela 2. Índice de Diversidade de Shannon $\left(\mathrm{H}^{\prime}\right)$ e Equabilidade de Pielou (J) em área em processo de recuperação no município de Abelardo Luz, estado de Santa Catarina, Brasil, para as avaliações realizadas em 2016 e 2017. *Valores não diferem entre si de acordo com o teste t de Hutcheson $(\mathrm{p}<0,05)$.

Table 2. Shannon Diversity Index $\left(H^{\prime}\right)$ and Pielou Equity $(J)$ in an area under recovery in the municipality of Abelardo Luz, state of Santa Catarina, Brazil, for the 2016 and 2017 assessments. * Values do not differ from each other. according to Hutcheson's $t$-test $(p<0.05)$.

\begin{tabular}{lccc}
\hline Levantamentos & Classe & Shannon $\left(\mathbf{H}^{\prime}\right)$ & Pielou (J) \\
\hline \multirow{3}{*}{ Avaliação 2016 } & Jovem & 1,63 & 0,67 \\
& Adulto & 1,70 & 0,87 \\
& Total & $\mathbf{1 , 7 5} \mathbf{a}^{*}$ & $\mathbf{0 , 6 8}$ \\
\hline \multirow{3}{*}{ Avaliação 2017 } & Jovem & 2,01 & 0,76 \\
& Adulto & 1,87 & 0,75 \\
& Total & $\mathbf{2 , 0 9} \mathbf{b}^{*}$ & $\mathbf{0 , 7 3}$ \\
\hline
\end{tabular}

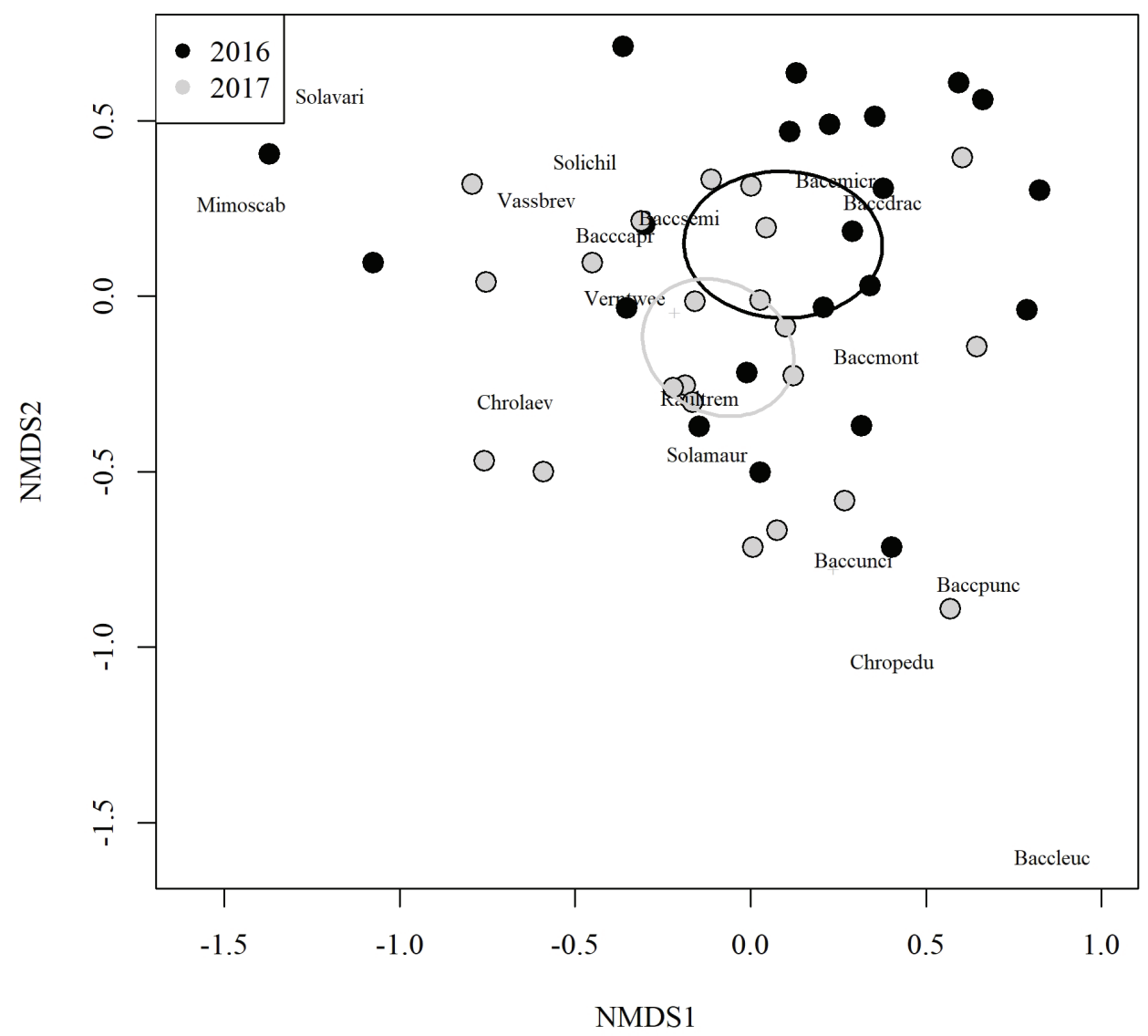

Figura 4. Ordenação de parcelas e das espécies amostradas em uma área de regeneração natural em processo de recuperação no município de Abelardo Luz, estado de Santa Catarina, Brasil, por meio da análise de Escalonamento Multidimensional Não-métrico (Stress $=0,17$ ). Elipses produzidas a partir do erro padrão dos centroides das parcelas agrupadas conforme os anos de 2016 (preto) e 2017 (cinza).

Figure 4. Ordering off plots and sampling in a natural regeneration area undergoing recovery in the municipality of Abelardo Luz, Santa Catarina State, Brazil, through the analysis of non-metric multidimensional scaling (Stress $=0.17$ ). Ellipses produced from the standard error of centroids of plots grouped according to the years 2016 (black) and 2017 (gray). 
Tabela 3. Classificação dos indivíduos regenerantes segundo a síndrome de dispersão para os dois estratos avaliados (jovens e adultos), nas duas avaliações realizadas (2016 e 2017), no município de Abelardo Luz, estado de Santa Catarina, Brasil. Valores entre parênteses são os valores esperados de acordo com o teste Qui-quadrado $(\mathrm{p}<0,05)$ e os valores fora dos parênteses são os valores observados no levantamento.

Table 3. Classification of regenerating individuals according to the dispersion syndrome for the two strata evaluated (young and adult), in the two evaluations, the first (2016 and 2017), in Abelardo Luz, state of Santa Catarina, Brazil. Values in parentheses are the expected values according to the chi-square test $(p<0.05)$ and the values outside the parenthesis are the values observed in the survey.

\begin{tabular}{lccc}
\hline Análise & Síndrome de Dispersão & Jovens & Adultos \\
\hline \multirow{2}{*}{2016} & Zoocórica & $1(9)$ & $11(3)$ \\
& Anemocórica & $200(190)$ & $46(56)$ \\
& Autocórica & $0(2)$ & $2(1)$ \\
\hline \multirow{2}{*}{2017} & Zoocórica & $7(19)$ & $27(14)$ \\
& Anemocórica & $286(271)$ & $186(201)$ \\
& Autocórica & $0(2)$ & $4(2)$ \\
\hline
\end{tabular}

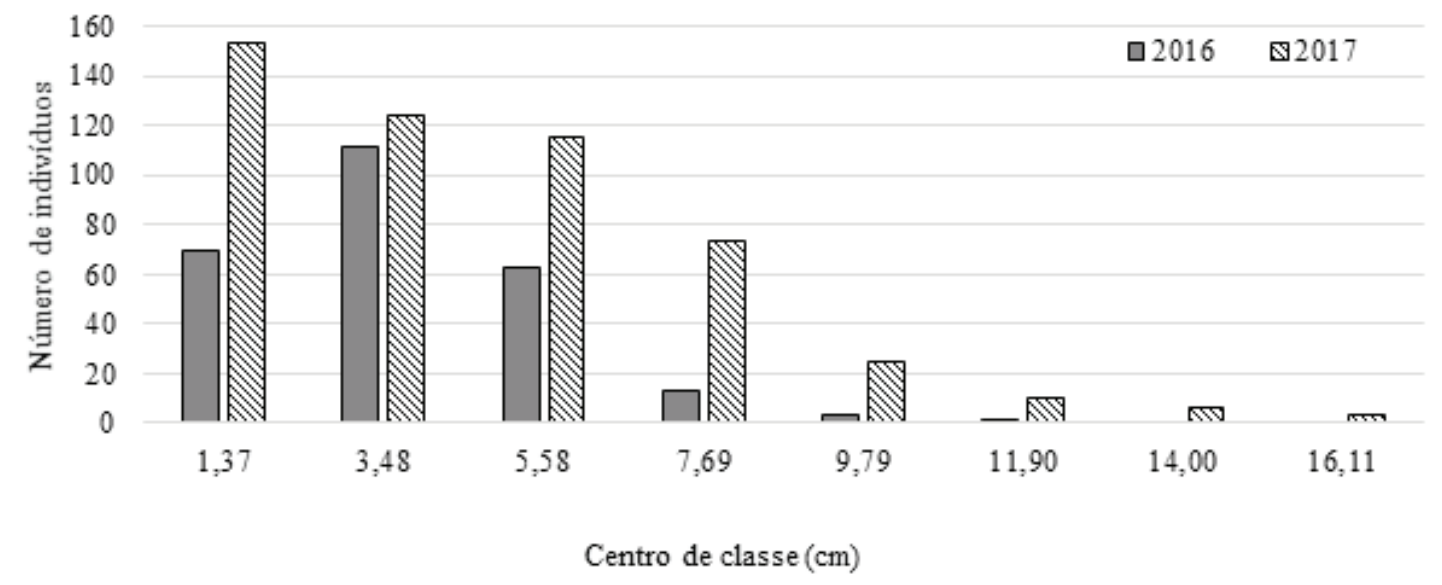

Figura 5. Distribuição do número de indivíduos em classes de diâmetro em área em processo de recuperação no município de Abelardo Luz, estado de Santa Catarina, Brasil.

Figure 5. Distribution of the number of individuals in diameter classes in an area under recovery in the municipality of Abelardo Luz, Santa Catarina state, Brazil.

diamétrica encontrado na segunda avaliação é também conhecido como modelo de distribuição "J invertido" ou exponencial negativa.

\section{DISCUSSÃO}

Os resultados indicaram uma comunidade em fase inicial de sucessão, com todas as espécies pioneiras, a maioria pertencente à família Asteraceae, e com predominância da síndrome de dispersão anemocórica síndrome de dispersão mais comum entre esse grupo sucessional. A elevada presença de indivíduos da família Asteraceae caracteriza o cenário das populações pioneiras e anemocóricas, pois normalmente é uma família com espécies de elevado potencial de adaptação ambiental, sendo consideradas espécies colonizadoras de ambientes degradados ou perturbados (Ferreira $e t$ $a l$. 2013) sendo que a síndromes de dispersão mais significativa na família é a anemocoria (Flora SBS 2019). Os resultados observados condizem com o histórico da área, pois no passado a área era um local destinado ao cultivo agrícola e pecuário, com matriz no entorno também de cultivo agrícola, o que provavelmente resultou em um banco de sementes antigo e persistente, característico desse grupo sucessional. Além disso, o pequeno espaço de tempo desde o desapropriamento da área e os levantamentos realizados, assim como o fato da área não receber outras atividades de recuperação além do revolvimento do solo e cercamento, podem ter contribuído para os padrões encontrados.

A ausência de espécies de outros grupos 
sucessionais na regeneração natural pode ser devido ao pouco tempo de avaliação, porém se a condição se prolongar, esta indica a possível necessidade de intervenções que possam suprir a área com espécies secundárias tardias e climáticas, utilizando-se técnica de enriquecimento. Venturoli et al. (2011) afirmam que os plantios de enriquecimentos visam melhorar a qualidade da regeneração, com a introdução de espécies nativas adequadas ao local. Mesmo assim, foi observado um aumento tanto no número de indivíduos quanto na riqueza e diversidade de espécies ao longo de um ano, sugerindo uma tendência ao avanço sucessional. O aumento da riqueza de espécies, a substituição de espécies iniciais por espécies de outros grupos sucessionais, e o aumento da disponibilidade de nichos são esperado quando maior o tempo de restauração de uma área (Rech et al. 2015). Ainda, povoamentos naturais com maior diversidade costumam ser mais eficientes nos processos de realibilitação de área degradadas (Cadotte et al. 2011).

Por se tratar de uma área com histórico de uso agrícola por longo período de tempo e com apenas dois anos de cercamento e início do processo de recuperação, os valores dos índices de Shannon (1,87 para os adultos e 2,01 para os jovens) e Pielou ( 0,75 para os adultos e 0,76 para os jovens) estão coerentes, já que as áreas agrícolas causam diversos impactos ao meio ambiente como o desmatamento, perda do banco de sementes do solo, erosão, perda da biodiversidade, poluição, geração de resíduos, entre outros danos (Bittencourt 2009, Deus \& Bakonyi 2012). O esperado é que, com maior tempo de recuperação, os valores dos índices aumentem.

Em um estudo prévio realizado também no estado de Santa Catarina, que avaliou estes índices seis anos após o início do processo de recuperação e que, diferente do presente estudo, também avaliou mudas que haviam sido plantadas, foram encontrados valores de $\mathrm{H}^{\prime}=2,91$ para os indivíduos adultos e $\mathrm{H}^{\prime}=3,5$ para os jovens, e $\mathrm{J}=0,78$ para os adultos e J =0,83 para jovens (Rech et al. 2015). Esse estudo ressalta a importância que a regeneração natural está desempenhando no local, pois além do aumento dos índices avaliados gerou a formação de novos nichos ecológicos, a cobertura do solo e favoreceu a recuperação do local com outras formas de vida (Rech etal. 2015). Portanto, um maior tempo de recuperação, junto da avaliação das áreas, pode gerar ainda melhorias nas características do local.

Em geral os valores de equabilidade encontrados indicaram uniformidade na distribuição dos indivíduos entre as espécies e baixa dominância. Porém, quando se observa que ocorreu uma diminuição no valor de equabilidade para os indivíduos adultos de 2016 para 2017, pode-se afirmar que no estrato arbóreo arbustivo adulto está ocorrendo o aumento na dominância por uma ou poucas espécies, sugerindo que poucas espécies estão encontrando condições ideais para a sua perpetuação no local.

Entre as espécies encontradas, destacamse aquelas observadas com maior número de indivíduos e típicas de locais antropizados, como, por exemplo, B. dracunculifolia, B. microdonta, Vernonanthura tweediana, Baccharis montana e Solanum mauritianum. Estas espécies são relatadas como tendo preferência por locais antropizados, como capoeirões, lavouras abandonadas, borda de rios e de florestas, locais abertos, pastagens, bordas de estradas e rodovias, chegando a formar grandes adensamentos em capoeiras, sendo espécies características como pioneiras antrópicas (Barroso \& Bueno 2002).

Os resultados encontrados pela ordenação através do NMDS, distância de Bray Curtis e NPMANOVA confirmam os padrões de mudança florístico-estrutural sugeridos anteriormente pelos aumentos no número de indivíduos, riqueza e diversidade, indicando que, embora aparentemente lento, o processo de colonização possa estar ocorrendo na área. Não foi possível verificar mudanças nos padrões de síndrome de dispersão ao longo do tempo, indicando que, mesmo havendo diferenças florístico-estruturais, as espécies estão sendo substituídas por outras também anemocóricas.

Os padrões de distribuição diamétrica também foram distintos entre os dois períodos de avaliação, sendo uma distribuição unimodal com forte assimetria positiva no primeiro inventário e um padrão "J invertido" no segundo. Uma distribuição unimodal indica que a regeneração da floresta se dá em ciclos e não de forma contínua, sendo característica de povoamentos jovens, encontrada eventualmente em floresta nativa (Téo et al. 2011). Já o modelo de distribuição "J invertido" é um modelo comum em florestas inequiâneas (i.e., florestas nativas não plantadas ou com indivíduos 
no mesmo povoamento de diferentes idades), caracterizado por maiores frequências nas classes de menor diâmetro e menores frequências nas maiores classes de diâmetro, sugerindo que as populações que compõem a comunidade são estáveis e que existe um balanço entre mortalidade e recrutamento de indivíduos (Souza et al. 2012). Assim, é possível inferir que a mudança de padrão diamétrico na área de estudo está seguindo uma direção que melhor representa uma floresta natural.

Considerando o exposto, com o passar do tempo foi evidenciado alterações no processo de recuperação que podem ser entendidos como avanços na colonização do local, que foram representadas no presente estudo pelo aumento no número de indivíduos, riqueza e diversidade, assim como na melhor representatividade da estrutura diamétrica. No entanto, considerando o processo de colonização lento e a falta de espécies de grupos sucessionais mais tardios, assim como o aumento na dominância dos indivíduos adultos, salienta-se a continuação da avaliação e acompanhamento do desenvolvimento da área e se necessário a efetivação de plantios de enriquecimento.

\section{AGRADECIMENTOS}

Gostaríamos de agradecer a Fundação de Amparo à Pesquisa de Santa Catarina (FAPESC) pela concessão da bolsa de Pós-Graduação (Mestrado). A Empresa de Consultoria GeoConsultores Engenharia e Meio Ambiente pela disponibilidade da oportunidade de desenvolver a pesquisa e auxilio de fomento à pesquisa. À Gabriel Lourenço Jesus, Carine Heinz Farias e Chaiane Rodrigues Schneider pelo apoio em campo.

\section{REFERÊNCIAS}

Alvares, C. A., Stape, J. L., Sentelhas, P. C., Moraes, G., Lonardo, J., \& Sparoveck, G. 2013. Köppen's climate classification map for Brazil. Meteorologische Zeitschrift, 22(6), 711-728. DOI: $10.1127 / 0941-2948 / 2013 / 0507$

Angiosperm Phylogeny Group. 2016. An update of the Angiosperm Phylogeny Group classification for the orders and families of flowering plants: APG IV. Botanical Journal of the Linnean Society, 181(2), 1-20. DOI: 10.1111/boj.12385
Barroso, G. M., \& Bueno, O. L. 2002. Subtribo: Baccharidinae. In: R. Reitz (Ed.), Flora ilustrada catarinense. pp. 765-1065. Itajaí, SC: Herbário Barbosa Rodrigues.

Bellotto, A., Viani, R. A. G., Nave, A. G., Gandolfi, S., \& Rodrigues. R. R. 2009. Monitoramento das áreas restauradas como ferramenta para avaliação da efetividade das ações de restauração e para redefinição metodológica. In: R. R. Rodrigues, P. H. S. Brancalion, \& I. Isernhagem (Eds.), Pacto pela restauração da Mata Atlântica: referencial dos conceitos e ações de restauração florestal. pp. 128-146. São Paulo: Instituto BioAtlântica.

Bittencourt, M. V. L. 2009. Impactos da agricultura no meio-ambiente: principais tendências e desafios (parte 1). Revista Economia \& Tecnologia, 18(5), 133-146. DOI: 10.5380/ret. v5i3.27144

Brancalion, P. H. S., Viani, R. A. G., Rodrigues, R. R., \& Gandolfi, S. 2012. Avaliação e monitoramento de áreas em processo de restauração. pp. 1-28. São Paulo: ESALQ.

Budowski, G. 1970. The distinction between old secondary and climax species in Tropical Central American lowland forests. Tropical Ecology, 11(1), 44-48.

Campos, J. B., \& Silveira-Filho, L. 2010. Floresta com Araucária. Volume 4. Série Ecossistemas Paranaenses: ACS/SEMA: p. 8.

Cadotte, M. W., Carscadden, K., \& Mirotchnick, N. 2011. Beyond species: functional diversity and the maintenance of ecological processes and services. Journal of Applied Ecology, 48(5), 10791087. DOI: $10.1111 /$ j.1365- 2664.2011.02048.x

CLIMATE - DATA. ORG. 2017. Clima: Abelardo Luz - SC. Acessado em 02/01/2018 em https:// pt.climate-data.org/location/43640/

Deus, R. M., \& Bakonyi, S. M. C. 2012. O impacto da agricultura sobre o meio ambiente. Revista Eletrônica em Gestão, Educação e Tecnologia Ambiental, 7(7), 1306-1315.

Ferreira, J. B. 2011. Estudo de impactos ambientais e medidas mitigadoras para uma obra de linhas de transmissão de energia elétrica. Trabalho de Conclusão de Curso Graduação em Agronomia. Departamento de Agronomia Universidade Federal de Santa Catarina. p. 48.

Ferreira, P. I., Gomes, J. P., Batista, F., Bernardi, A. P., Costa, N. C. F, Bortoluzzi, R. L., \& Mantovani, A. 2013. Espécies potenciais para recuperação de 
áreas de preservação permanente no planalto catarinense. Floresta e Ambiente, 20(2), 173182. DOI: 10.4322 /floram.2013.003

Flora SBS. 2019. Asteraceae. Acessado em 19/08/2019 em https://sites.google.com/site/ florasbs/asteraceae

Hauresko, C., Correia. R. L., \& Gomes, M. F. V. B. 2017. A relação entre a conservação ambiental da floresta com araucárias e os sistemas faxinais no Paraná. Revista Pegada, 18(1), 131-151.

Kageyama, P., \& Gandara, F. B. 2000. Recuperação de áreas ciliares. In: R. R. Rodrigues, \& H. F. Leitão-Filho (Eds.), Matas Ciliares: Conservação e recuperação. pp. 320. São Paulo, SP: EDUSP/ FAPESP

Klein, R.M. 1978. Mapa fitogeográfico do estado de Santa Catarina. In: R. Reitz (Ed.), Flora ilustrada catarinense. p. 24. Itajaí, SC: Herbário Barbosa Rodrigues.

Ministério do Meio Ambiente. 2015. A floresta com Araucárias. Acessado em 19/12/2018 em http:/ / www.mma.gov.br/estruturas/202/_arquivos/ folder_consulta02.pdf

Oksanen, J., Blanchet, F. B., Friendly, M., Kindt, R., Legendre, P., McGlinn, D., Minchin P. R., O'Hara, R. B., Simpson, G. L., Solymos, P., Stevens, M. H.H., Szoecs, E., \& Wagner, H. 2018. Vegan: community ecology package. R package version, $1,8-8$.

Pereira, A. L. A. B. 2014. Análise crítica dos impactos ambientais ocasionados pela linha de transmissão $500 \mathrm{kV}$ Miracema-Sapeaçu e subestações associadas. Trabalho de Conclusão de curso. Departamento de engenharia ambiental da Escola Politécnica da Universidade Federal do Rio de Janeiro. p. 124.

Pereira, J. A. A., Oliveira-Filho, A. T., Eisenlohr, P. V., Miranda, P. L. S., \& Lemos-Filho, J. P. 2015. Human impacts affect tree community features of 20 forest fragments of a vanishing Neotropical hotspot. Environmental Management, 55(2), 296-307. DOI: 10.1007/s00267-014-0387-7

Portal ICMBio. 2019. Flora. Acessado em 21/08/2019 em http://www.icmbio.gov.br/parnaiguacu/ biodiversidade/18-flora2.html

Pires, L. F. A. 2005. Gestão ambiental da implantação de sistemas de transmissão de energia elétrica. Estudo de caso: Interligação Norte/Sul I. Dissertação de Mestrado em Ciência Ambiental. Departamento de Ciência Ambiental da
Universidade Federal Fluminense. p. 142.

Ribeiro, M. C., Metzger, J. P., Martensen, A. C., Ponzoni, F. J., \& Hirota, M. M. 2009. The Brazilian Atlantic Forest: how much is left, and how is the remaining for distributed? Implications for conservation. Biological Conservation, 142(6), 1141-1153. DOI: 10.1016/j. biocon.2009.02.021

R Development Core Team. 2018. R: A language and environment for statistical computing. $\mathrm{R}$ Foundation for Statistical Computing. Acessado em 05/05/2018 em http://www.Rproject.org

Rech, C. C. C., Silva, A. C., Higuchi, P., Schimalski, M. B., Pscheidt, F., Schmidt, A. B., Ansolin. R. J., Bento. M. A., Missio, F. F., \& Loebens, R. 2015. Avaliação da restauração florestal de uma APP degradada em Santa Catarina. Floresta e Ambiente, 22(2), 194-203. DOI: 10.1590/21798087.083414

Santana, I. 2018. Por uma compensação florestal inteligente em Brasília. Brasília: Correio Braziliense, Empresa Brasileira de Pesquisa Agropecuária.

Santos, V. S., Batista, A. P. B., Aparício, P. S., Aparício, W. C. S., \& Guedes, A. C. L. 2012. Dinâmica florestal de espécies arbóreas em uma floresta de várzea na cidade de Macapá, AP, Brasil. Revista Verde, 7(4), 207-213.

Schroter, C., \& Kirchner, O. 1902. Die vegetation des Bodensees. Zweiter Teil. Lindau, Germany: Stettner-Verlag: p. 80.

Souza, P. B., Souza, A. L., \& Meira Neto, J. A. A. 2012. Estrutura diamétrica dos estratos e grupos ecológicos de uma área de Floresta Estacional Semidecidual, em Dionísio, MG. Revista Árvore, 36(1), 151-160. DOI: 10.1590/S010067622012000100016

Téo, S. J., Rocha S. P., Bortoncello, A. C., Paz, R. A., \& Costa, R. H. 2011. Dinâmica da distribuição diamétrica de povoamentos de Pinus taeda, na região de Caçador, SC. Pesquisa Florestal Brasileira, 31(67), 183-192. DOI: 10.4336/2011. pfb.31.67.183

Tobouti, A. K., \& Santos, V. L. P. 2014. Impactos ambientais causados na implantação de linhas de transmissão no Brasil. Caderno Meio Ambiente e Sustentabilidade, 4(3), 184 - 199.

Turmina, E., Kanieski, M. R., Jesus, L. A., Rosa, L. H., Batista, L. G., \& Almeida, A. N. 2018. Avaliação de impactos ambientais gerados na implantação e operação de subestação de energia elétrica: 
100 | Regeneração natural em área de recuperação

um estudo de caso em Palhoça, SC. Revista de Ciências Agroveterinárias, 17(4), 589 - 598. DOI: 10.5965/223811711732018589

Van Der Pijl, L. 1982. Principles of dispersal in higher plants. Berlim: Springer-Verlag: p. 197.

Venturoli, F., Fagg, C. W., \& Felfili, M. 2011. Desenvolvimento inicial de Dipteryx alata Vogel e Myracrodruon urundeuva Allemão em plantio de enriquecimento de uma floresta estacional semidecídua secundária. Bioscience Journal, 27(3), 482-493.

Submitted: 05 November 2018

Accepted: 27 August 2019

Published online: 03 September 2019

Associate Editor: Nuria Pistón 